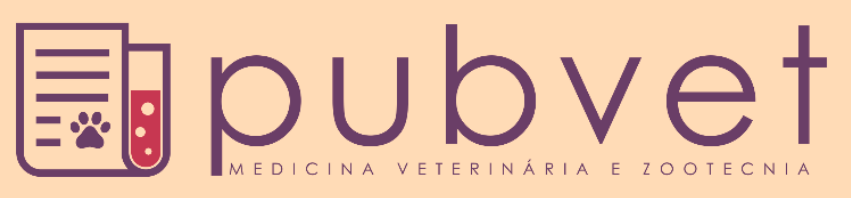

https://doi.org/10.31533/pubvet.v13n10a434.1-15

\title{
Indicadores de eficiência econômica e os principais índices zootécnicos encontrados em propriedades leiteiras na Região Norte Fluminense
}

\author{
Fernanda Giácomo Ragazzi ${ }^{1 *}$, Douglas do Couto Mena $^{2} \bullet$, Victor Cruz Rodrigues ${ }^{3} \bullet$, Carlos \\ Augusto de Oliveira ${ }^{3}$, Afonso Aurélio de Carvalho Peres ${ }^{4}{ }^{\circ}$ \\ ${ }^{I}$ Discente do Programa de Pós-Graduação da Universidade Federal Rural do Rio de Janeiro; \\ ${ }^{2}$ Discente do Programa de Pós-Graduação da Universidade Federal do Rio Grande do Sul \\ ${ }^{3}$ Professor do Departamento de Produção e Avaliação Animal da Universidade Federal Rural do Rio de Janeiro \\ ${ }^{4}$ Professor do Departamento de Engenharia de Agronegócios da VEA/Universidade Federal Fluminense \\ *Autor para correspondência, E-mail: ragazzi.fernanda@gmail.com
}

Resumo. Objetivou-se analisar os índices zootécnicos: taxa de natalidade, intervalo de partos, período de serviço e produção de leite, os indicadores de eficiência econômica: margem bruta (MB), margem líquida (ML) e resultado (RE) - (lucro ou prejuízo) além da composição dos custos de produção de 20 propriedades leiteiras na região Norte Fluminense. As propriedades leiteiras obtiveram as seguintes médias para os índices zootécnicos; taxa de natalidade de 57,4 $\pm 2,18 \%$ com coeficiente de variação de $21,22 \%$, intervalo de partos de $13,5 \pm 0,68$ meses com coeficiente de variação de 5,05\%, período de serviço de 116,2 \pm 9,23 dias com coeficiente de variação de 7,95\% e produção de leite de $6,1 \pm 2,58$ litros por dia com coeficiente de variação de $41,96 \%$. Foi encontrada MB média de $\mathrm{R} \$ 102.731,82 \pm \mathrm{R} \$ 68.087,24$ com coeficiente de variação de $66,28 \%$ e ML média de $\mathrm{R} \$ 93.531,22 \pm \mathrm{R} \$ 67.948,36$ com coeficiente de variação de $72,65 \%$, permitindo aos produtores permanecerem na atividade no curto prazo. E RE (prejuízo) na média de - R $\$$ $360.809,02 \pm \mathrm{R} \$ 89.067,61 \mathrm{com}$ coeficiente de variação de 24,69\%. Por apresentar prejuízo, a receita obtida não remunera o investimento realizado. Os baixos índices zootécnicos referentes à taxa de natalidade e produção de leite devem ser melhorados para obtenção de maiores receitas na atividade e melhor produção de leite. A maior porcentagem de participação na composição das receitas e nas despesas de investimento foi o valor imobilizado, quando contabilizado, e a venda do leite e a compra de animais, respectivamente, quando não contabilizado, $o$ valor imobilizado. Com relação às despesas com custeio, as maiores porcentagens foram referentes à mão de obra, a pastagem, a alimentação concentrada e a suplementação volumosa. Os produtores analisados permanecem no curto prazo na atividade, porém para aumentar a produção devem ter melhorados os índices zootécnicos com maior utilização da assistência técnica para obtenção de maior retorno de eficiência econômica.

Palavras-chave: Custo de produção, indicadores econômicos, índices zootécnicos

\section{Economic efficiency indicators and the main zootechnical indices found in dairy properties in the North Fluminense Region}

Abstract. The objective to analyze the animal indexes: birth rate, calving intervals, period of service and production of milk, and the indicators of economic efficiency: gross margin $(\mathrm{GM})$, net margin (NM) and results (RE) - (profit or loss), in addition to the composition of the costs of production of 20 dairy properties in the fluminense north region. The dairy properties obtained the following average for the zootechnical indexes: birth rates of 57,4 \pm $2,18 \%$ with the coefficient of variation of $21,22 \%$, calving intervals of $13,5 \pm 0,68$ months 
with coefficient of variation of 7,95\% and milk production of $6,1 \pm 2,58$ liters per day with coefficient of variation of $41,96 \%$. It was found GM average of $\mathrm{R} \$ 102.731,82 \pm \mathrm{R} \$$ $68.087,24$ with coefficient of variation of $66,28 \%$ e and $N M$ average of $R \$ 93.531,22 \pm R \$$ $67.948,36$ with coefficient of variation of $72,65 \%$, allowing the producers to remain in the activity on a short term. And $\mathrm{RE}$ (loss) average of - $\mathrm{R} \$ 360.809,02 \pm \mathrm{R} \$ 89.067,61$ with coefficient of variation of $24,69 \%$. Because it showed loss, the revenue obtained does not remunerate the investment made. The low zootechnical indexes referring to birth rates and milk production must be improved for obtaining larger revenues in the activity better milk production. The highest percentage of participation in the composition of revenue and in investment expenditure was the asset value, when accounted, and the milk sell and animal purchase, respectively, when not accounted, the asset value. Regarding the expenditure with payment of costs, the higher percentages were related to labor, pasture, concentrated feed and bulky supplementation. The producers analyzed remained in the activity for a short term, however, to increase production they must have improved the zootechnical indexes with greater use of technical assistance to obtain a higher return on economic efficiency.

Key words: Cost of production, economic indicators, animal indexes

\title{
Indicadores de eficiencia económica y los principales índices zootécnicos encontrados en las propiedades lácteas de la región norte de Fluminense
}

\begin{abstract}
Resumen. El objetivo fue analizar los índices zootécnicos: tasa de natalidad, intervalo de partos, período de servicio y producción de leche, los indicadores de eficiencia económica: margen bruto $(\mathrm{MB})$, margen neto $(\mathrm{NM})$ y resultado $(\mathrm{RE})$ - (beneficio o pérdida) y la composición de los costos de producción de 20 granjas lecheras en la región del norte de Fluminense. Las granjas lecheras obtuvieron los siguientes promedios para los índices zootécnicos; tasa de natalidad de $57.4 \pm 2.18 \%$ con un coeficiente de variación del $21.22 \%$, intervalo de parto de $13.5 \pm 0.68$ meses con un coeficiente de variación de $5.05 \%$, período de servicio de $116.2 \pm 9.23$ días con un coeficiente de variación del $7.95 \%$ y producción de leche de $6.1 \pm 2.58$ litros por día con un coeficiente de variación del $41.96 \%$. Encontramos un MB promedio de $\mathrm{R} \$ 102,731.82 \pm \mathrm{R} \$ 68,087.24$ con un coeficiente de variación del $66.28 \%$ y un NM promedio de $\mathrm{R} \$ 93,531.22 \pm \mathrm{R} \$ 67,948.36$ con un coeficiente de variación del $72.65 \%$, permitiendo a los productores permanecer en el negocio a corto plazo. Y RE (pérdida) promediada - R \$360,809.02 $\pm \mathrm{R} \$ 89,067.61$ con un coeficiente de variación del $24.69 \%$. Como presenta pérdidas, los ingresos obtenidos no remuneran la inversión realizada. Los bajos índices zootécnicos relacionados con la tasa de natalidad y la producción de leche deben mejorarse para obtener mayores ingresos por actividad y una mejor producción de leche. El mayor porcentaje de participación en la composición de los ingresos y gastos de inversión fue el valor fijo, cuando se contabilizó, y la venta de leche y la compra de animales, respectivamente, cuando no se contabilizaron, el valor fijo. En cuanto a los gastos de costos, los porcentajes más altos se relacionaron con mano de obra, pasto, alimentación concentrada y suplementos. Los productores analizados permanecen en el corto plazo en la actividad, pero para aumentar la producción deben haber mejorado los índices zootécnicos con un mayor uso de asistencia técnica para obtener un mayor retorno de la eficiencia económica.
\end{abstract}

Palabras clave: Costo de producción, indicadores económicos. índices zootécnicos

\section{Introdução}

Diversas transformações na produção de leite ao longo dos anos têm contribuído para que os produtores reflitam sobre a realidade de se administrar a atividade tornando-se mais eficientes e competitivos. Entretanto, muitos produtores não têm conseguido gerir suas propriedades com a eficiência necessárias para mantê-los em situação competitiva no mercado. Nesse contexto, o acompanhamento de indicadores zootécnicos e econômicos pode ser utilizado como ferramenta para o gerenciamento do desempenho técnico-econômico da atividade leiteira que auxiliem nos ganhos 
produtivos (Pereira et al., 2010). Dados sobre os custos de produção na pecuária leiteira, segundo Lopes \& Carvalho (2000) são utilizados para a verificação de como estão sendo empregados os recursos disponíveis no processo produtivo do leite. Informações sobre o custo de produção também são utilizadas para cálculos dos valores econômicos para produções leiteiras para analisar a quantidade desejada de lucro por unidade melhorada por conta da gestão dos custos (Bueno et al., 2004; Madalena et al., 2001; Martins, 2004). Por conta disso, o controle estratégico e a gestão da propriedade tornam-se cada vez mais importantes no dia a dia dos produtores que estão inseridos neste mercado com expectativas de permanência neste setor.

Pesquisas a campo têm sido realizadas em todo o território nacional em propriedades rurais de diversos tamanhos, seja onde o leite é a atividade principal ou a atividade secundária como renda, para auxiliar o produtor em assuntos relacionados aos custos de produção e aos fatores que interferem na atividade (Pereira et al., 2010; Robbs \& Campelo, 2002). Contudo, são necessários estudos regionalizados já que essa atividade sofre alterações devido aos fatores climáticos, diversidade socioeconômica e competitividade dos mercados lácteos encontrada em cada região brasileira.

O objetivo deste estudo foi realizar a análise de custos de produção de vinte propriedades leiteiras localizadas na região Norte Fluminense em relação aos índices zootécnicos do rebanho: taxa de natalidade, intervalo de partos, período de serviço e produção de leite, os componentes dos custos de produção: custo operacional efetivo, custo operacional total e custo total de produção e os indicadores de eficiência econômica: margem bruta, margem líquida e resultado (lucro ou prejuízo).

\section{Material e métodos}

\section{Obtenção e caracterização dos produtores}

O trabalho foi realizado em 20 propriedades rurais vinculadas a cooperativa de laticínios localizada na região Norte Fluminense, entre os meses de janeiro e dezembro de 2002 e atualizados pelo IGP-DI para agosto de 2016. A coleta de dados deu-se em visitas a campo para as anotações das informações de rotina diárias e mensais das propriedades, que após consolidadas, foram digitalizadas e armazenadas em coletor eletrônico desenvolvido no programa MS-Excel ${ }^{\circledR}$. O grupo caracterizou-se por médios produtores segundo classificação de Lopes et al. (2005), com aproximadamente 50 hectares destinados à produção de leite, com criações extensivas a pasto e com produções de cana-de açúcar. As pastagens cultivadas representaram o principal suporte alimentar dos rebanhos. Os rebanhos eram formados por mestiços de Holandês vs. Zebu, havendo predominância no grau genético do Zebu a raça Gir, com a reprodução ocorrendo através da monta natural a campo, sem controle zootécnico e com animais de variados grupamentos genéticos. Sendo ainda pouco adotadas práticas de inseminação artificial e cruzamentos dirigidos ao melhoramento genético. Em todas as propriedades o leite produzido foi destinado à cooperativa, sendo o transporte realizado por caminhão tanque.

\section{Organização das tabelas avaliativas}

Os dados de interesse no trabalho foram organizados em planilhas, sendo adotadas as seguintes categorias como componentes dos custos de produção: Mão de obra contratada: valores pagos aos funcionários das respectivas propriedades, além de acrescida de $80 \%$ dos direitos previstos nas leis trabalhistas. Pastagem: valores de implantação e manutenção como, análise de solo, hora máquina para aração e gradagem do solo, plantio de sementes, aplicação de corretivos e fertilizantes. Alimentação concentrada: valores gastos com alimentação comercial do rebanho. Sal mineral: referentes à aquisição de mistura mineral comercial. Assistência técnica: valores pagos ao técnico responsável pelas atividades da propriedade, sendo esta, disponibilizada pela própria cooperativa a baixo custo. Depreciação: referente às benfeitorias, máquinas e equipamentos. Animais: vacas em lactação e vacas secas, machos reprodutores, fêmeas de 1 a 2 anos, novilhas, bezerras e bezerros constituintes dos rebanhos. Valor imobilizado: em terra e animais para produção (vacas leiteiras e machos reprodutores), utilizados no primeiro ano de investimento. Suplementação volumosa: incluindo polpa cítrica, cana-de-açúcar, capim picado, cevada e silagem. Despesas diversas: valores pagos ao frete do leite, taxas e impostos variáveis em função da produção. Medicamentos em geral: todos os produtos de uso veterinário, destinados à sanidade do rebanho. Luz e telefone: relacionados aos gastos com energia elétrica e rede telefônica disponível na propriedade. Reparos e manutenção do sistema de produção: despesas que não podem ser 
evitadas, mesmo que a produção seja alterada. São necessárias para que o bem não tenha seu valor comprometido, como conservação de cercas e telhados, manutenção das instalações entre outros.

\section{Indicadores zootécnicos de interesse do trabalho}

Foram usados os seguintes índices: taxa de natalidade, intervalo de partos, período de serviço e produção de leite. Para a taxa de natalidade utilizou-se o total de vacas em lactação dividido pelo total de vacas do rebanho (secas e lactantes), obtendo-se as porcentagens da taxa de natalidade dos 20 rebanhos analisados, variando de 0 a $100 \%$ de nascimentos nos rebanhos em função do total das vacas. Foram anotados os valores de intervalo de partos em meses e o período de serviço em dias, utilizando os dados dos rebanhos analisados.

Para a obtenção da produção de leite em litros por vaca por dia (L/v/d) foi utilizado o controle leiteiro de cada propriedade dividido pelas vacas em lactação e por 30 dias. Após obtenção desses valores, foram montadas tabelas para melhor visualização e compreensão dos resultados apresentando também a média, o desvio padrão da média e o coeficiente de variação.

\section{Componentes do custo de produção avaliados}

Custo fixo: proveniente do capital fixo das propriedades analisadas. Custo variável: custos que variaram de acordo com a quantidade produzida e que possuíram duração igual a um ciclo de produção (Aguiar \& Almeida, 2002; Lopes \& Carvalho, 2000).

Custo de oportunidade ou alternativo: custo que o fator de produção possuiu devido ao seu uso na atividade leiteira e não a sua utilização em outra atividade alternativa no mercado. Para o cálculo deste custo, foram considerados os valores referentes à depreciação e aos animais de produção (vacas leiteiras e os reprodutores) multiplicados pela taxa de $6 \%$ ao ano, referentes aos valores da poupança de acordo com o Portal Brasil.

Custo operacional efetivo: custos onde ocorreram efetivamente desembolsos ou dispêndios em dinheiro por parte dos produtores (Lopes \& Lopes, 2001). Custo operacional total: soma do custo operacional efetivo e outros custos como a depreciação de bens, máquinas e equipamentos (Lopes \& Lopes, 2001). Custo total: todo o custo que a propriedade possuía relacionado à atividade leiteira.

\section{Indicadores de eficiência econômica em propriedades rurais}

Margem bruta: medida de resultado econômico, de acordo com Lopes \& Lopes (2001), considerando que o produtor possui recursos disponíveis e precisa tomar decisões de forma eficaz quanto aos fatores de produção envolvidos (terra, capital e trabalho). A matemática foi calculada pela seguinte fórmula:

Margem bruta $(\mathrm{MB})=$ receita bruta $(\mathrm{RB})$ - custos operacionais efetivos $(\mathrm{COE})$

Margem líquida: permitiu analisar se a atividade estava estável, e se poderia ocorrer expansões na produção quando pensada em longo prazo. Matematicamente, para Lopes \& Lopes (2001), a margem líquida pode ser calculada com a seguinte fórmula:

Margem líquida $(\mathrm{ML})=$ receita bruta $(\mathrm{RB})$ - custo operacional total $(\mathrm{COT})$

Resultado: diferença entre as receitas e os custos totais. Apresenta-se positivo quando a atividade cobre custos totais, representando lucro, negativo quando não são pagos os custos totais, representando prejuízo ou então quando a receita se iguala aos custos totais indicando estabilidade na produção com tendência de investimentos em longo prazo. Matematicamente, pode ser calculado com a seguinte fórmula:

Resultado = receita bruta $(\mathrm{RB})-$ custo total $(\mathrm{CT})$

\section{Resultados e discussão}

\section{Análise comparativa dos índices zootécnicos}

As taxas de natalidade encontradas apresentaram média de 57,4 $\pm 12,18 \%$ com coeficiente de variação de 21,22\% (Tabela 1). Sugerindo que a média apresentada não foi satisfatória para a produção de leite, por ser este um indicador relacionado à lucratividade dos sistemas de produção. As taxas de 
natalidade dos rebanhos variaram de 77,0 até 31,9\%. Pereira et al. (2009) trabalhando com diferentes rebanhos na região de Guaratinguetá/SP encontraram taxa de natalidade média de 81,6\%, demonstrando ser este valor um bom indicador zootécnico para os rebanhos mestiços na região do Vale do Paraíba/SP.

Em relação ao intervalo de partos (Tabela 1), encontrou-se média de 13,5 $\pm 0,68$ meses, com coeficiente de variação de 5,05\%. Pereira et al. (2009) encontraram valores de intervalo de partos próximos aos encontrados neste trabalho, com 13,3 meses. Estes resultados são inferiores aos 18-19 meses citado como a média da maioria dos rebanhos leiteiros do Brasil (Ferreira, 2002; Pereira et al., 2009). Entretanto, como o intervalo de partos torna-se uma informação limitada por causa da inclusão de matrizes com diversas idades e número de partos distintos, o período de serviço para os rebanhos foi calculado, sendo encontrada a média de 116,2 \pm 9,23 dias com coeficiente de variação de 7,95\% (Tabela 1).

De acordo com a literatura, o ideal é um período de serviço de 85 a 120 dias e a gestação de nove meses, compreendendo um parto por ano ou um parto a cada treze meses e esses produtores, quando analisados em relação à média, conseguiram obter este resultado.

Tabela 1. Indicadores zootécnicos: taxa de natalidade, intervalo de parto, período de serviço e produção de leite de 20 propriedades leiteiras na região Norte Fluminense

\begin{tabular}{lcccc}
\hline Produtor & Taxa de natalidade $(\%)$ & Intervalo de partos (meses) & Período de serviço (dias) & Produção de leite (litros) \\
\hline 01 & 76,3 & 13,8 & 122,0 & 4,2 \\
02 & 57,3 & 13,8 & 125,0 & 4,7 \\
03 & 77,0 & 12,7 & 98,0 & 8,5 \\
04 & 53,3 & 14,2 & 121,2 & 4,9 \\
05 & 56,6 & 14,1 & 124,3 & 4,7 \\
06 & 32,8 & 14,5 & 128,0 & 5,9 \\
07 & 61,0 & 13,1 & 114,2 & 3,8 \\
08 & 61,3 & 13,4 & 121,2 & 3,1 \\
09 & 60,7 & 13,0 & 108,1 & 4,5 \\
10 & 53,2 & 14,1 & 119,0 & 3,2 \\
11 & 50,1 & 13,5 & 116,0 & 4,2 \\
12 & 54,3 & 14,2 & 123,5 & 4,4 \\
13 & 52,8 & 12,9 & 103,6 & 5,9 \\
14 & 49,7 & 13,6 & 123,5 & 7,0 \\
15 & 61,3 & 12,8 & 116,5 & 11,0 \\
16 & 73,7 & 12,7 & 104,3 & 7,3 \\
17 & 65,7 & 12,5 & 103,9 & 5,9 \\
18 & 48,4 & 13,9 & 124,5 & 11,2 \\
19 & 70,7 & 12,3 & 103,2 & 11,3 \\
20 & 31,9 & 14,3 & 123,5 & 7,2 \\
\hline Média & 57,4 & 13,5 & 116,2 & 6,1 \\
D.P. & 12,18 & 0,68 & 9,23 & $41,96 \%$ \\
\hline V. & $21,22 \%$ & $5,05 \%$ & $7,95 \%$ & \\
\hline
\end{tabular}

Fatores ligados ao desenvolvimento e aplicação de práticas como a inseminação artificial voltada para melhoria das condições genéticas do rebanho, adoção de práticas de higiene na ordenha e de limpeza e desinfecção das instalações da propriedade leiteira podem melhorar a produtividade dos rebanhos analisados.

Ainda em relação aos indicadores zootécnicos, a produção de leite em litros encontrada foi na média $6,1 \pm 2,56$ litros com coeficiente de variação de $41,96 \%$. Bueno (2013) encontrou em seus resultados a média de produção de leite de 14,5 litros por vaca em rebanhos mestiços, trabalhando com sistema exclusivo a pasto com utilização de forrageiras tropicais associadas com forragens de inverno. Entretanto, dados divulgados pela FAERJ (2010) mostram que a produção de leite no Estado do Rio de Janeiro, está em torno de 6,67 litros por vaca por dia, estando estes produtores de um modo geral, bem próximos deste perfil. Contudo, neste grupo de produtores, existem propriedades com produções muito 
baixas e outra com maiores produções, alcançando valores de 3,0 a 6,0 litros por vaca e 8,5 a 11,0 litros por vaca, respectivamente. As baixas produções de leite acabam ocasionando perdas econômicas para os produtores uma vez que os mesmos têm dispêndios com alimentação concentrada, pastagem e suplementação volumosa sem o retorno esperado na produção de leite (Godinho \& Carvalho, 2013).

Para estes produtores, de modo geral, a produção de leite exclusivamente a pasto poderá representar um sistema mais econômico e viável utilizando apenas suplementação na entressafra (outono-inverno) de forma a equilibrar a alimentação. Dados publicados por Deresz (1994) mostram produções de leite diárias de 12 a $14 \mathrm{~kg}$, usando vacas mestiças Holandesas vs. Zebus em pastejo rotativo sem a utilização de concentrado, confirmando que pode ser uma alternativa a ser utilizada nestes casos. Em estudos realizados por Silva et al. (2008), os sistemas mais dependentes de pastagem apresentaram melhores resultados econômicos, com menores gastos na alimentação e retorno na produção de leite. Além de investir em aquisição de animais com bons índices produtivos e boas produções de leite, que também pode ser o caminho para aumentar a produção de leite.

\section{Avaliação dos resultados econômicos}

As propriedades apresentaram resultados favoráveis à atividade obtendo variações monetárias em função das produções inerentes a cada propriedade (Tabela 2), descontado o valor do capital imobilizado.

Tabela 2. Resultados econômicos: total de receitas, total de investimento e total de custeio de 20 propriedades leiteiras na região Norte Fluminense, em Reais

\begin{tabular}{|c|c|c|c|}
\hline Propriedades & Receitas & Investimentos & Custeio \\
\hline$\overline{1}$ & $\mathrm{R} \$ 384.640,49$ & $\mathrm{R} \$ 68.760,39$ & $\mathrm{R} \$ 73.420,89$ \\
\hline 2 & $\mathrm{R} \$ 278.539,89$ & $\mathrm{R} \$ 39.796,03$ & $\mathrm{R} \$ 209.201,72$ \\
\hline 3 & $\mathrm{R} \$ 145.845,91$ & $\mathrm{R} \$ 14.472,35$ & $\mathrm{R} \$ 43.812,64$ \\
\hline 4 & $\mathrm{R} \$ 247.031,44$ & $\mathrm{R} \$ 23.024,47$ & $\mathrm{R} \$ 156.473,02$ \\
\hline 5 & $\mathrm{R} \$ 128.242,95$ & $\mathrm{R} \$ 32.532,42$ & $\mathrm{R} \$ 84.749,52$ \\
\hline 6 & $\mathrm{R} \$ 295.392,72$ & $\mathrm{R} \$ 27.493,03$ & $\mathrm{R} \$ 118.504,66$ \\
\hline 7 & $\mathrm{R} \$ 136.269,41$ & $\mathrm{R} \$ 37.327,72$ & $\mathrm{R} \$ 51.622,42$ \\
\hline 8 & $\mathrm{R} \$ 445.784,32$ & $\mathrm{R} \$ 37.691,44$ & $\mathrm{R} \$ 201.102,80$ \\
\hline 9 & $\mathrm{R} \$ 239.127,61$ & $\mathrm{R} \$ 19.915,49$ & $\mathrm{R} \$ 97.275,26$ \\
\hline 10 & $\mathrm{R} \$ 228.416,82$ & $\mathrm{R} \$ 16.448,79$ & $\mathrm{R} \$ 136.254,45$ \\
\hline 11 & $\mathrm{R} \$ 295.678,42$ & $\mathrm{R} \$ 12.629,67$ & $\mathrm{R} \$ 156.036,43$ \\
\hline 12 & $\mathrm{R} \$ 199.575,53$ & $\mathrm{R} \$ 17.258,17$ & $\mathrm{R} \$ 82.862,67$ \\
\hline 13 & $\mathrm{R} \$ 244.249,61$ & $\mathrm{R} \$ 22.022,40$ & $\mathrm{R} \$ 132.306,81$ \\
\hline 14 & $\mathrm{R} \$ 223.200,12$ & $\mathrm{R} \$ 19.956,43$ & $\mathrm{R} \$ 63.027,30$ \\
\hline 15 & $\mathrm{R} \$ 350.865,51$ & $\mathrm{R} \$ 19.520,54$ & $\mathrm{R} \$ 143.959,34$ \\
\hline 16 & $\mathrm{R} \$ 257.230,02$ & $\mathrm{R} \$ 17.869,17$ & $\mathrm{R} \$ 118.244,18$ \\
\hline 17 & $\mathrm{R} \$ 152.225,02$ & $\mathrm{R} \$ 23.824,38$ & $\mathrm{R} \$ 91.525,96$ \\
\hline 18 & $\mathrm{R} \$ 203.811,33$ & $\mathrm{R} \$ 21.421,98$ & $\mathrm{R} \$ 158.274,98$ \\
\hline 19 & $\mathrm{R} \$ 110.661,02$ & $\mathrm{R} \$ 8.277,66$ & $\mathrm{R} \$ 97.893,81$ \\
\hline 20 & $\mathrm{R} \$ 172.172,39$ & $\mathrm{R} \$ 25.171,49$ & $\mathrm{R} \$ 125.188,11$ \\
\hline Média & $\mathrm{R} \$ 236.948,03$ & $\mathrm{R} \$ 25.270,70$ & $\mathrm{R} \$ 117.086,85$ \\
\hline DP & $\mathrm{R} \$ 88.228,25$ & $\mathrm{R} \$ 13.266,75$ & $\mathrm{R} \$ 45.776,27$ \\
\hline $\mathrm{CV}$ & 37,24 & 52,50 & 39,1 \\
\hline
\end{tabular}

Em relação à receita média obtida, os produtores podem ser classificados como médios produtores de acordo com a escala de produção proposta pelo mesmo autor.

Foi encontrada receita total média de $\mathrm{R} \$ 81.283,97 \pm \mathrm{R} \$ 28.693,43$ com coeficiente de variação de $35,30 \%$. A composição da receita (Tabela 8) foi formada, em média, pela venda do leite que representou $77,72 \pm 12,06 \%$ da composição e pela venda de animais, que representou $22,28 \pm 12,06 \%$ nesta composição. Lopes et al. (2006) encontraram valores de receitas para propriedades com pequenas, médias e grandes escalas de produções de $\mathrm{R} \$ 24.665,60, \mathrm{R} \$ 62.588,08$ e $\mathrm{R} \$ 357.668,07$ e composição da receita de soma dos valores apurados com a venda de leite 79,09\%, 91,99\% e 90,31\% e animais 
$20,29 \%, 7,26 \%$ e $9,70 \%$, estando os produtores analisados distribuídos dentro deste perfil de escala de produção.

\section{Composição dos itens de avaliação dos sistemas de produção}

Os valores de investimentos relacionados à aquisição de animais (bezerros e bezerras, fêmeas, novilhas e machos para reprodução), permitem a produção conjunta de alguns animais, pois os produtores compram bezerros para revenda, além do valor de depreciação (Tabela 2) referente aos bens utilizados na atividade, onde foi encontrada média de $\mathrm{R} \$ 25.270,70 \pm \mathrm{R} \$ 13.266,75$ com coeficiente de variação de 52,50\%.

Com relação aos valores de custeios na atividade, encontrou-se média de $\mathrm{R} \$ 117.086,85 \pm \mathrm{R} \$$ 45.776,27 com coeficiente de variação de 39,10\%.

A diferença entre os valores gastos com investimentos e custeios podem estar relacionados com a quantidade de animais de cada rebanho, pois maiores aquisições de animais vão necessitar de maior dispêndio financeiro e maior uso dos insumos referentes ao dia a dia da atividade. Os resultados econômicos relacionados aos valores monetários de depreciação, custo de oportunidade do capital estável e custo de oportunidade do capital circulante são apresentados a seguir (Tabela 3).

Tabela 3. Resultados econômicos: depreciação (DEP), custo de oportunidade do capital estável (COp.est.) e custo de oportunidade do capital circulante (COp.circ.) de 20 propriedades leiteiras na região Norte Fluminense, em Reais

\begin{tabular}{lccc}
\hline Propriedades & $\mathrm{DEP}$ & $\mathrm{COp} . \mathrm{est}$ & $\mathrm{COp.circ.}$ \\
\hline 1 & $\mathrm{R} \$ 9.154,77$ & $\mathrm{R} \$ 24.488,26$ & $\mathrm{R} \$ 3.990,80$ \\
2 & $\mathrm{R} \$ 9.143,04$ & $\mathrm{R} \$ 12.844,01$ & $\mathrm{R} \$ 7.166,40$ \\
3 & $\mathrm{R} \$ 9.119,81$ & $\mathrm{R} \$ 9.827,88$ & $\mathrm{R} \$ 2.112,90$ \\
4 & $\mathrm{R} \$ 9.345,94$ & $\mathrm{R} \$ 12.977,15$ & $\mathrm{R} \$ 5.104,55$ \\
5 & $\mathrm{R} \$ 9.864,63$ & $\mathrm{R} \$ 10.769,04$ & $\mathrm{R} \$ 3.222,52$ \\
6 & $\mathrm{R} \$ 9.273,43$ & $\mathrm{R} \$ 15.710,13$ & $\mathrm{R} \$ 3.954,41$ \\
7 & $\mathrm{R} \$ 8.822,59$ & $\mathrm{R} \$ 11.203,42$ & $\mathrm{R} \$ 2.403,83$ \\
8 & $\mathrm{R} \$ 10.002,13$ & $\mathrm{R} \$ 21.158,00$ & $\mathrm{R} \$ 6.863,76$ \\
9 & $\mathrm{R} \$ 9.044,55$ & $\mathrm{R} \$ 11.348,22$ & $\mathrm{R} \$ 3.244,39$ \\
10 & $\mathrm{R} \$ 9.348,52$ & $\mathrm{R} \$ 16.289,31$ & $\mathrm{R} \$ 4.300,64$ \\
11 & $\mathrm{R} \$ 9.498,20$ & $\mathrm{R} \$ 17.954,43$ & $\mathrm{R} \$ 4.775,04$ \\
12 & $\mathrm{R} \$ 9.011,42$ & $\mathrm{R} \$ 11.782,60$ & $\mathrm{R} \$ 2.733,28$ \\
13 & $\mathrm{R} \$ 9.173,37$ & $\mathrm{R} \$ 17.501,95$ & $\mathrm{R} \$ 4.354,68$ \\
14 & $\mathrm{R} \$ 9.202,84$ & $\mathrm{R} \$ 13.845,91$ & $\mathrm{R} \$ 2.387,59$ \\
15 & $\mathrm{R} \$ 9.348,44$ & $\mathrm{R} \$ 14.352,69$ & $\mathrm{R} \$ 4.623,94$ \\
16 & $\mathrm{R} \$ 9.114,87$ & $\mathrm{R} \$ 13.737,31$ & $\mathrm{R} \$ 3.809,95$ \\
17 & $\mathrm{R} \$ 9.609,57$ & $\mathrm{R} \$ 11.818,80$ & $\mathrm{R} \$ 3.172,22$ \\
18 & $\mathrm{R} \$ 8.740,54$ & $\mathrm{R} \$ 11.710,20$ & $\mathrm{R} \$ 5.128,69$ \\
19 & $\mathrm{R} \$ 8.277,66$ & $\mathrm{R} \$ 10.045,07$ & $\mathrm{R} \$ 2.936,81$ \\
20 & $\mathrm{R} \$ 8.915,71$ & $\mathrm{R} \$ 15.438,64$ & $\mathrm{R} \$ 4.243,32$ \\
\hline Média & $\mathrm{R} \$ 9.200,60$ & $\mathrm{R} \$ 14.240,15$ & $\mathrm{R} \$ 4.026,49$ \\
$\mathrm{CP}$ & $\mathrm{R} \$ 382,31$ & $\mathrm{R} \$ 3.798,07$ & 33,86 \\
\hline
\end{tabular}

Para as 20 propriedades leiteiras analisadas, observou-se que a depreciação obtida com média de $\mathrm{R} \$$ $9.200,60 \pm \mathrm{R} \$ 382,31$ e com coeficiente de variação de 4,16\%. O valor obtido demonstrou semelhança entre as propriedades no que se refere à depreciação de bens sendo necessária a capitalização financeira pelos produtores ao longo do tempo com a finalidade de substituição do patrimônio, após sua vida útil, permitindo a continuidade da atividade leiteira.

Com relação aos custos de oportunidade do capital estável e custo de oportunidade do capital circulante, foram encontrados os seguintes valores médios para as propriedades: $R \$ 14.240,15 \pm R \$$ $3.798,07$ com coeficiente de variação $26,67 \%$ e média de $\mathrm{R} \$ 4.026,49 \pm \mathrm{R} \$ 1.363,34$ com coeficiente de variação de 33,86 , respectivamente. 
Para o custo de oportunidade do capital circulante foram utilizados os valores de mão de obra permanente, assistência técnica e custos variáveis, ou seja, os itens que estão diretamente relacionados com a atividade no dia a dia, que são efetivamente ligados à produção. Sendo positivos para a produção de leite.

Foi encontrada para o custo operacional efetivo (Tabela 4) a média de $\mathrm{R} \$ 133.835,80 \pm \mathrm{R} \$ 46.657,23$ com coeficiente de variação de $34,86 \%$. Este resultado sugere que os produtores se mantêm no curto prazo porque o custo operacional efetivo está sendo coberto pela receita obtida, servindo para orientar decisões administrativas de curto prazo (Tabela 4). Em relação ao custo operacional total (Tabela 4), foi encontrada a média de $\mathrm{R} \$ 143.051,40 \pm \mathrm{R} \$ 46.791,89$ e coeficiente de variação de $32,71 \%$, ou seja, os produtores cobrem os gastos com custo operacional efetivo necessário para a atividade, além do custo com a depreciação.

Tabela 4. Resultados econômicos: custo operacional efetivo (COE), custo operacional total (COT) e custo total (CT) de 20 propriedades leiteiras na região Norte Fluminense, em Reais

\begin{tabular}{lccc}
\hline Propriedades & $\mathrm{COE}$ & $\mathrm{COT}$ & $\mathrm{CT}$ \\
\hline 01 & $\mathrm{R} \$ 133.026,51$ & $\mathrm{R} \$ 142.181,28$ & $\mathrm{R} \$ 551.827,15$ \\
02 & $\mathrm{R} \$ 238.880,14$ & $\mathrm{R} \$ 248.023,17$ & $\mathrm{R} \$ 676.156,97$ \\
03 & $\mathrm{R} \$ 70.429,93$ & $\mathrm{R} \$ 79.549,74$ & $\mathrm{R} \$ 407.145,77$ \\
04 & $\mathrm{R} \$ 170.151,55$ & $\mathrm{R} \$ 179.497,49$ & $\mathrm{R} \$ 612.069,05$ \\
05 & $\mathrm{R} \$ 107.417,31$ & $\mathrm{R} \$ 117.281,94$ & $\mathrm{R} \$ 476.249,98$ \\
06 & $\mathrm{R} \$ 131.813,78$ & $\mathrm{R} \$ 141.087,21$ & $\mathrm{R} \$ 664.758,22$ \\
07 & $\mathrm{R} \$ 80.127,54$ & $\mathrm{R} \$ 88.950,13$ & $\mathrm{R} \$ 462.397,55$ \\
08 & $\mathrm{R} \$ 228.792,12$ & $\mathrm{R} \$ 238.794,25$ & $\mathrm{R} \$ 944.060,85$ \\
09 & $\mathrm{R} \$ 108.146,19$ & $\mathrm{R} \$ 117.190,75$ & $\mathrm{R} \$ 495.464,62$ \\
10 & $\mathrm{R} \$ 143.354,72$ & $\mathrm{R} \$ 152.703,25$ & $\mathrm{R} \$ 695.680,10$ \\
11 & $\mathrm{R} \$ 159.167,90$ & $\mathrm{R} \$ 168.666,10$ & $\mathrm{R} \$ 767.147,26$ \\
12 & $\mathrm{R} \$ 91.109,42$ & $\mathrm{R} \$ 100.120,84$ & $\mathrm{R} \$ 492.874,10$ \\
13 & $\mathrm{R} \$ 145.155,85$ & $\mathrm{R} \$ 154.329,21$ & $\mathrm{R} \$ 737.727,68$ \\
14 & $\mathrm{R} \$ 79.586,32$ & $\mathrm{R} \$ 88.789,17$ & $\mathrm{R} \$ 550.319,49$ \\
15 & $\mathrm{R} \$ 154.131,44$ & $\mathrm{R} \$ 163.479,88$ & $\mathrm{R} \$ 641.902,82$ \\
16 & $\mathrm{R} \$ 126.998,47$ & $\mathrm{R} \$ 136.113,34$ & $\mathrm{R} \$ 594.023,82$ \\
17 & $\mathrm{R} \$ 105.740,77$ & $\mathrm{R} \$ 115.350,34$ & $\mathrm{R} \$ 509.310,21$ \\
18 & $\mathrm{R} \$ 170.956,43$ & $\mathrm{R} \$ 179.696,97$ & $\mathrm{R} \$ 570.037,00$ \\
19 & $\mathrm{R} \$ 97.893,81$ & $\mathrm{R} \$ 106.171,47$ & $\mathrm{R} \$ 441.007,20$ \\
20 & $\mathrm{R} \$ 141.443,89$ & $\mathrm{R} \$ 150.359,60$ & $\mathrm{R} \$ 664.981,00$ \\
\hline Média & $\mathrm{R} \$ 133.835,80$ & $\mathrm{R} \$ 46.791,89$ & 22,40 \\
$\mathrm{DP}$ & $\mathrm{R} \$ 46.657,23$ & 32,71 & $\mathrm{R} \$ 133.076,87$ \\
$\mathrm{CV}$ & 34,86 & & 218,94 \\
\hline
\end{tabular}

Para diminuir o custo operacional total (COT) podem ser utilizadas duas alternativas, o aumento da eficiência e a produção em escala (Lopes et al., 2006). De acordo com Gomes \& Alves (1999), que compararam a eficiência de produtores de leite, poderia ser obtida uma redução de $43 \%$ no custo operacional total de produtores ineficientes, produzindo-se a mesma quantidade de leite. Quanto ao aumento na escala, esse pode ser conseguido através do aumento do rebanho total e, por consequência, do rebanho em lactação ou da produtividade por animal.

\section{Indicadores de eficiência econômica}

Como indicadores de eficiência econômica foram determinados a margem bruta (Tabela 5), margem líquida (Tabela 6) e o resultado (Tabela 7) dos sistemas de produção de leite analisados.

Para a margem bruta, foi encontrada média de $\mathrm{R} \$ 102.731,82 \pm \mathrm{R} \$ 68.087,24$ com coeficiente de variação de 66,28\%, média de margem bruta por animal de $\mathrm{R} \$ 1.504,75 \pm \mathrm{R} \$ 1.083,66$ com coeficiente de variação de $72,02 \%$ e margem bruta por área na média de $\mathrm{R} \$ 2.323,74 \pm \mathrm{R} \$ 1.567,57$ com coeficiente de variação de $67,46 \%$ para os sistemas analisados. 
Tabela 5. Indicador de eficiência econômica: margem bruta (MB), por animal e por área, de 20 propriedades leiteiras na região Norte Fluminense, em Reais

\begin{tabular}{|c|c|c|c|}
\hline Propriedades & MB & MB/animal & MB/área \\
\hline$\overline{01}$ & $\mathrm{R} \$ 251.613,98$ & $\mathrm{R} \$ 4.493,11$ & $\mathrm{R} \$ 5.591,42$ \\
\hline 02 & $\mathrm{R} \$ 39.659,76$ & $\mathrm{R} \$ 440,66$ & $\mathrm{R} \$ 881,33$ \\
\hline 03 & $\mathrm{R} \$ 75.415,99$ & $\mathrm{R} \$ 3.428,00$ & $\mathrm{R} \$ 1.675,91$ \\
\hline 04 & $\mathrm{R} \$ 76.879,89$ & $\mathrm{R} \$ 985,64$ & $\mathrm{R} \$ 1.922,00$ \\
\hline 05 & $\mathrm{R} \$ 20.825,64$ & $\mathrm{R} \$ 595,02$ & $\mathrm{R} \$ 462,79$ \\
\hline 06 & $\mathrm{R} \$ 163.578,95$ & $\mathrm{R} \$ 1.460,53$ & $\mathrm{R} \$ 3.635,09$ \\
\hline 07 & $\mathrm{R} \$ 56.141,87$ & $\mathrm{R} \$ 1.369,31$ & $\mathrm{R} \$ 1.247,60$ \\
\hline 08 & $\mathrm{R} \$ 216.992,20$ & $\mathrm{R} \$ 2.169,92$ & $\mathrm{R} \$ 5.424,81$ \\
\hline 09 & $\mathrm{R} \$ 130.981,41$ & $\mathrm{R} \$ 2.786,84$ & $\mathrm{R} \$ 2.910,70$ \\
\hline 10 & $\mathrm{R} \$ 85.062,10$ & $\mathrm{R} \$ 766,33$ & $\mathrm{R} \$ 1.890,27$ \\
\hline 11 & $\mathrm{R} \$ 136.510,53$ & $\mathrm{R} \$ 1.074,89$ & $\mathrm{R} \$ 3.033,57$ \\
\hline 12 & $\mathrm{R} \$ 108.466,11$ & $\mathrm{R} \$ 1.807,77$ & $\mathrm{R} \$ 2.410,36$ \\
\hline 13 & $\mathrm{R} \$ 99.093,76$ & $\mathrm{R} \$ 805,64$ & $\mathrm{R} \$ 2.202,08$ \\
\hline 14 & $\mathrm{R} \$ 143.613,79$ & $\mathrm{R} \$ 1.889,66$ & $\mathrm{R} \$ 3.191,42$ \\
\hline 15 & $\mathrm{R} \$ 196.734,07$ & $\mathrm{R} \$ 2.028,19$ & $\mathrm{R} \$ 4.371,87$ \\
\hline 16 & $\mathrm{R} \$ 130.231,55$ & $\mathrm{R} \$ 1.691,32$ & $\mathrm{R} \$ 2.894,03$ \\
\hline 17 & $\mathrm{R} \$ 46.484,25$ & $\mathrm{R} \$ 830,08$ & $\mathrm{R} \$ 1.032,98$ \\
\hline 18 & $\mathrm{R} \$ 32.854,90$ & $\mathrm{R} \$ 619,90$ & $\mathrm{R} \$ 730,11$ \\
\hline 19 & $\mathrm{R} \$ 12.767,21$ & $\mathrm{R} \$ 510,69$ & $\mathrm{R} \$ 283,72$ \\
\hline 20 & $\mathrm{R} \$ 30.728,50$ & $\mathrm{R} \$ 341,43$ & $\mathrm{R} \$ 682,86$ \\
\hline Média & $\mathrm{R} \$ 102.731,82$ & $\mathrm{R} \$ 1.504,75$ & $\mathrm{R} \$ 2.323,74$ \\
\hline DP & $\mathrm{R} \$ 68.087,24$ & $\mathrm{R} \$ 1.083,66$ & $\mathrm{R} \$ 1.567,57$ \\
\hline $\mathrm{CV}$ & $\mathrm{R} \$ 66,28$ & $\mathrm{R} \$ 72,02$ & $\mathrm{R} \$ 67,46$ \\
\hline
\end{tabular}

Tabela 6. Indicador de eficiência econômica: margem líquida (ML), por animal e por área, de 20 propriedades leiteiras na região Norte Fluminense, em Reais

\begin{tabular}{|c|c|c|c|}
\hline Propriedades & ML & ML/animal & ML/área \\
\hline$\overline{01}$ & $\mathrm{R} \$ 242.459,21$ & $\mathrm{R} \$ 4.329,63$ & $\mathrm{R} \$ 5.387,98$ \\
\hline 02 & $\mathrm{R} \$ 30.516,72$ & $\mathrm{R} \$ 339,07$ & $\mathrm{R} \$ 678,15$ \\
\hline 03 & $\mathrm{R} \$ 66.296,18$ & $\mathrm{R} \$ 3.013,46$ & $\mathrm{R} \$ 1.473,25$ \\
\hline 04 & $\mathrm{R} \$ 67.533,95$ & $\mathrm{R} \$ 865,82$ & $\mathrm{R} \$ 1.688,35$ \\
\hline 05 & $\mathrm{R} \$ 10.961,01$ & $\mathrm{R} \$ 313,17$ & $\mathrm{R} \$ 243,58$ \\
\hline 06 & $\mathrm{R} \$ 154.305,51$ & $\mathrm{R} \$ 1.377,73$ & $\mathrm{R} \$ 3.429,01$ \\
\hline 07 & $\mathrm{R} \$ 47.319,28$ & $\mathrm{R} \$ 1.154,13$ & $\mathrm{R} \$ 1.051,54$ \\
\hline 08 & $\mathrm{R} \$ 206.990,07$ & $\mathrm{R} \$ 2.069,90$ & $\mathrm{R} \$ 5.174,75$ \\
\hline 09 & $\mathrm{R} \$ 121.936,86$ & $\mathrm{R} \$ 2.594,40$ & $\mathrm{R} \$ 2.709,71$ \\
\hline 10 & $\mathrm{R} \$ 75.713,58$ & $\mathrm{R} \$ 682,10$ & $\mathrm{R} \$ 1.682,52$ \\
\hline 11 & $\mathrm{R} \$ 127.012,33$ & $\mathrm{R} \$ 1.000,10$ & $\mathrm{R} \$ 2.822,50$ \\
\hline 12 & $\mathrm{R} \$ 99.454,69$ & $\mathrm{R} \$ 1.657,58$ & $\mathrm{R} \$ 2.210,10$ \\
\hline 13 & $\mathrm{R} \$ 89.920,39$ & $\mathrm{R} \$ 731,06$ & $\mathrm{R} \$ 1.998,23$ \\
\hline 14 & $\mathrm{R} \$ 134.410,95$ & $\mathrm{R} \$ 1.768,57$ & $\mathrm{R} \$ 2.986,91$ \\
\hline 15 & $\mathrm{R} \$ 187.385,63$ & $\mathrm{R} \$ 1.931,81$ & $\mathrm{R} \$ 4.164,13$ \\
\hline 16 & $\mathrm{R} \$ 121.116,67$ & $\mathrm{R} \$ 1.572,94$ & $\mathrm{R} \$ 2.691,48$ \\
\hline 17 & $\mathrm{R} \$ 36.874,68$ & $\mathrm{R} \$ 658,48$ & $\mathrm{R} \$ 819,44$ \\
\hline 18 & $\mathrm{R} \$ 24.114,36$ & $\mathrm{R} \$ 454,99$ & $\mathrm{R} \$ 535,87$ \\
\hline 19 & $\mathrm{R} \$ 4.489,55$ & $\mathrm{R} \$ 179,58$ & $\mathrm{R} \$ 99,77$ \\
\hline 20 & $\mathrm{R} \$ 21.812,79$ & $\mathrm{R} \$ 242,36$ & $\mathrm{R} \$ 484,73$ \\
\hline Média & $\mathrm{R} \$ 93.531,22$ & $\mathrm{R} \$ 1.346,84$ & $\mathrm{R} \$ 2.116,60$ \\
\hline DP & $\mathrm{R} \$ 67.948,36$ & $\mathrm{R} \$ 1.065,44$ & $\mathrm{R} \$ 1.561,70$ \\
\hline $\mathrm{CV}$ & $\mathrm{R} \$ 72,65$ & $\mathrm{R} \$ 79,11$ & $\mathrm{R} \$ 73,78$ \\
\hline
\end{tabular}


Estes resultados apresentaram-se superiores aos encontrados por Gonçalves (2013), no qual foram encontrados valores de margem bruta de $\mathrm{R} \$ 45.574,92$, margem bruta por animal de $\mathrm{R} \$ 759,58 \mathrm{e}$ margem bruta por área de $\mathrm{R} \$ 3.038,32$.

Foi observada baixa eficiência produtiva nos rebanhos dos produtores analisados neste trabalho, contudo, o valor positivo obtido para a margem bruta permite concluir que a atividade leiteira está se remunerando e pode sobreviver, pelo menos no curto prazo.

Em relação aos indicadores: margem líquida, margem líquida por animal e margem líquida por área, os resultados apresentaram-se positivos na atividade (Tabela 6). O indicador de eficiência econômica (Tabela 7) apresentou-se negativo, representando prejuízo na atividade.

Tabela 7. Indicador de eficiência econômica: resultado de 20 propriedades leiteiras na região Norte Fluminense, em Reais

\begin{tabular}{lc}
\hline Propriedade & Resultado \\
\hline 01 & $-\mathrm{R} \$ 167.186,66$ \\
02 & $-\mathrm{R} \$ 397.617,07$ \\
03 & $-\mathrm{R} \$ 261.299,86$ \\
04 & $-\mathrm{R} \$ 365.037,61$ \\
05 & $-\mathrm{R} \$ 348.007,03$ \\
06 & $-\mathrm{R} \$ 369.365,50$ \\
07 & $-\mathrm{R} \$ 326.128,14$ \\
08 & $-\mathrm{R} \$ 498.276,53$ \\
09 & $-\mathrm{R} \$ 256.337,02$ \\
10 & $-\mathrm{R} \$ 467.263,28$ \\
11 & $-\mathrm{R} \$ 471.468,83$ \\
12 & $-\mathrm{R} \$ 293.298,57$ \\
13 & $-\mathrm{R} \$ 493.478,08$ \\
14 & $-\mathrm{R} \$ 327.119,38$ \\
15 & $-\mathrm{R} \$ 291.037,31$ \\
16 & $-\mathrm{R} \$ 336.793,81$ \\
17 & $-\mathrm{R} \$ 357.085,20$ \\
18 & $-\mathrm{R} \$ 366.225,67$ \\
19 & $-\mathrm{R} \$ 330.346,18$ \\
20 & $-\mathrm{R} \$ 492.808,61$ \\
\hline Média & $-\mathrm{R} \$ 360.809,02$ \\
DP & $\mathrm{R} \$ 89.067,61$ \\
$\mathrm{CV}$ & 24,69 \\
\hline
\end{tabular}

Com relação ao indicador de eficiência econômica, Resultado na atividade leiteira foi obtido para os sistemas de produção analisados, a média de $-\mathrm{R} \$ 360.809,02 \pm \mathrm{R} \$$ 89.067,61 com coeficiente de variação de 24,69\%, compreendendo prejuízo na atividade analisada. Deste modo a expansão da atividade só será possível caso os indicadores de eficiência econômica permaneçam positivos e associados a melhorias nos indicadores zootécnicos de produção.

\section{Composição dos itens de avaliação dos sistemas de produção}

Com relação à receita obtida através da atividade leiteira relacionada aos produtores analisados, foram verificados os itens que compõem a receita sendo separados em venda do leite e venda de animais, bem como estes mesmos itens acrescidos do valor imobilizado (terra, vacas leiteiras e reprodutores), em comparação com a composição sem o valor imobilizado (Tabela 8). O valor imobilizado novamente mencionado foi considerado como despesa no primeiro mês de análise e como receita no final dos doze meses de observação. Com relação à composição da receita, sem considerar o valor imobilizado, composta apenas pela venda do leite e pela venda de animais, foi encontrada a média de 78,10 $\pm 12,75 \%$ com coeficiente de variação de 16,33\%. Estes valores foram semelhantes aos resultados encontrados por Lopes et al. (2009) com valores para a venda do leite médio de $86,94 \%$ da composição da receita dos produtores. 
Para a venda de animais, foi encontrado como porcentagem de composição da receita, a média de $21,90 \pm 12,75 \%$ com coeficiente de variação de $58,23 \%$, sendo superiores aos encontrados por Almeida Júnior et al. (2002) estudando o efeito da venda de animais na rentabilidade de um sistema de produção de leite, com obtenção de valores médios de 16,95\% e por Lopes et al. (2004), Lopes et al. (2005), Lopes et al. (2006) e Lopes et al. (2009) que encontraram para a venda de animais valores médios de 10,16\%, $12,40 \%, 12,41 \%$ e $12,45 \%$ da receita da atividade leiteira, respectivamente.

Quando analisada a composição da receita considerando o valor imobilizado foi encontrada média composta por $30,37 \pm 8,04 \%$ com coeficiente de variação de $26,47 \%$ referente à venda do leite, $8,27 \pm$ $4,86 \%$ com coeficiente de variação de $58,76 \%$ referente à venda de animais e $61,36 \pm 6,43 \%$ com coeficiente de variação de $10,48 \%$ referente ao valor imobilizado.

Com relação à composição das despesas de investimento, relacionada às despesas com depreciação e compra de animais, o perfil entre os produtores apresentou-se semelhante entre os mesmos tendo inclusão ou não do valor imobilizado na atividade (Tabela 9).

Tabela 8. Composição das receitas obtidas de 20 propriedades leiteiras na região Norte Fluminense, com ou sem capital imobilizado, em porcentagem

\begin{tabular}{|c|c|c|c|c|c|}
\hline \multirow{2}{*}{ Propriedade } & \multicolumn{2}{|c|}{ Sem valor imobilizado (\%) } & \multicolumn{3}{|c|}{ Com valor imobilizado (\%) } \\
\hline & Venda de leite & Venda de animais & Venda de leite & Venda de animais & Imobilizado \\
\hline$\overline{01}$ & 92,03 & 7,97 & 49,91 & 4,32 & 45,77 \\
\hline 02 & 87,41 & 12,59 & 36,75 & 5,29 & 57,96 \\
\hline 03 & 95,42 & 4,58 & 34,32 & 1,65 & 64,03 \\
\hline 04 & 83,71 & 16,29 & 35,06 & 6,82 & 58,12 \\
\hline 05 & 70,18 & 29,82 & 21,81 & 9,27 & 68,93 \\
\hline 06 & 71,65 & 28,35 & 29,79 & 11,79 & 58,42 \\
\hline 07 & 69,64 & 30,36 & 21,96 & 9,57 & 68,47 \\
\hline 08 & 72,76 & 27,24 & 32,28 & 12,09 & 55,63 \\
\hline 09 & 53,27 & 46,73 & 23,64 & 20,74 & 55,63 \\
\hline 10 & 82,42 & 17,58 & 28,58 & 6,10 & 65,33 \\
\hline 11 & 89,74 & 10,26 & 34,46 & 3,94 & 61,60 \\
\hline 12 & 72,73 & 27,27 & 28,41 & 10,65 & 60,93 \\
\hline 13 & 86,67 & 13,33 & 29,96 & 4,61 & 65,43 \\
\hline 14 & 58,00 & 42,00 & 21,98 & 15,91 & 62,10 \\
\hline 15 & 91,11 & 8,89 & 43,79 & 4,27 & 51,94 \\
\hline 16 & 78,00 & 22,00 & 32,36 & 9,12 & 58,52 \\
\hline 17 & 67,64 & 32,36 & 22,17 & 10,61 & 67,23 \\
\hline 18 & 92,52 & 7,48 & 36,74 & 2,97 & 60,28 \\
\hline 19 & 88,88 & 11,12 & 26,16 & 3,27 & 70,57 \\
\hline 20 & 58,28 & 41,72 & 17,30 & 12,38 & 70,32 \\
\hline Média & 78,10 & 21,90 & 30,37 & 8,27 & 61,36 \\
\hline DP & 12,75 & 12,75 & 8,04 & 4,86 & 6,43 \\
\hline $\mathrm{CV}$ & 16,33 & 58,23 & 26,47 & 58,76 & 10,48 \\
\hline
\end{tabular}

Foi encontrado valor médio para a composição do item depreciação de 44,35 $\pm 19,58 \%$ com coeficiente de variação de $44,14 \%$ e para compra de animais, o valor médio de 55,65 $\pm 19,58 \%$ com coeficiente de variação de $35,18 \%$. E quando acrescido o valor imobilizado, os produtores apresentaram a composição das despesas com média de $1,97 \pm 0,36 \%$ com coeficiente de variação de $18,01 \%$ referente à depreciação, $3,76 \pm 3,38 \%$ com coeficiente de variação de $89,82 \%$ referente à compra de animais e $92,72 \pm 9,16 \%$ com coeficiente de variação de $9,88 \%$ referente ao valor imobilizado. Semelhante à composição das receitas, quando o valor imobilizado foi acrescentado na composição do item avaliado, por ser alto no primeiro ano, apresenta-se com maior participação no montante analisado. 
Tabela 9. Composição das despesas de investimento de 20 propriedades leiteiras na região Norte Fluminense, com ou sem capital imobilizado, em porcentagem

\begin{tabular}{|c|c|c|c|c|c|}
\hline \multirow{2}{*}{ Propriedade } & \multicolumn{2}{|c|}{ Sem valor imobilizado (\%) } & \multicolumn{3}{|c|}{ Com valor imobilizado (\%) } \\
\hline & Depreciação & Compra de animais & Depreciação & Compra de animais & Imobilizado \\
\hline 01 & 13,31 & 86,69 & 1,91 & 12,46 & 85,63 \\
\hline 02 & 22,97 & 77,03 & 1,74 & 5,85 & 92,41 \\
\hline 03 & 63,02 & 36,98 & 2,67 & 1,56 & 95,77 \\
\hline 04 & 40,59 & 59,41 & 2,05 & 3,00 & 94,95 \\
\hline 05 & 30,32 & 69,68 & 2,52 & 5,79 & 91,69 \\
\hline 06 & 33,73 & 66,27 & 1,68 & 3,31 & 95,01 \\
\hline 07 & 23,64 & 76,36 & 2,15 & 6,94 & 90,91 \\
\hline 08 & 26,54 & 73,46 & 1,35 & 12,09 & 55,63 \\
\hline 09 & 45,41 & 54,59 & 2,27 & 2,73 & 95,00 \\
\hline 10 & 56,83 & 43,17 & 1,67 & 1,27 & 97,06 \\
\hline 11 & 75,21 & 24,79 & 1,55 & 0,51 & 97,93 \\
\hline 12 & 52,22 & 47,78 & 2,20 & 2,01 & 95,79 \\
\hline 13 & 41,65 & 58,35 & 1,52 & 2,12 & 96,36 \\
\hline 14 & 46,11 & 53,89 & 1,91 & 2,23 & 95,86 \\
\hline 15 & 47,89 & 52,11 & 1,88 & 2,04 & 96,08 \\
\hline 16 & 51,01 & 48,99 & 1,92 & 1,84 & 96,24 \\
\hline 17 & 40,34 & 59,66 & 2,30 & 3,40 & 94,30 \\
\hline 18 & 40,80 & 59,20 & 2,12 & 3,08 & 94,80 \\
\hline 19 & 100,00 & 0,00 & 2,41 & 0,00 & 97,59 \\
\hline 20 & 35,42 & 64,58 & 1,65 & 3,01 & 95,34 \\
\hline Média & 44,35 & 55,65 & 1,97 & 3,76 & 92,72 \\
\hline DP & 19,58 & 19,58 & 0,36 & 3,38 & 9,16 \\
\hline $\mathrm{CV}$ & 44,14 & 35,18 & 18,01 & 89,82 & 9,88 \\
\hline
\end{tabular}

No que diz respeito à composição das despesas de custeio (Tabela 10), foi detalhada a participação de cada item em relação à maior ou menor contribuição apresentada no custo de produção. A mão de obra, que os produtores possuem em seus sistemas produtivos, apresentou a maior participação na composição das despesas com custeio com média de 33,68 \pm 8,57\% com coeficiente de variação de $25,4 \%$. Sendo superior aos resultados encontrados por Prado et al. (2007), que encontraram valores para mão de obra onerando os custos para produção em $29,60 \%$ sendo o item de maior impacto na atividade. E superiores aos valores encontrados por Lopes et al. (2009), onde a mão de obra correspondeu apenas a $13,37 \%$ do custeio na atividade leiteira.

A pastagem correspondeu a $26,88 \pm 11,24 \%$ com coeficiente de variação de $41,82 \%$ na composição do custeio para atividade, por estarem incluídos os valores de adubação, limpeza, plantio, compra de sementes, manutenção, entre outros, acabando por aumentar os gastos com este item. Diferente dos resultados encontrados por Gonçalves (2013), onde a pastagem representou apenas 9,90\% da composição do custeio, sendo apenas computados gastos com a manutenção.

A alimentação concentrada utilizada pelos produtores é a ração comercial, além da polpa cítrica encontrada a baixo custo na região, correspondendo a valores médios de 14,39 $\pm 8,58 \%$ com coeficiente de variação de 59,6\% na composição do custeio. Resultados superiores foram encontrados por Gonçalves (2013) com média de alimentação em 19,90\% de participação no custeio e por Rocha (2013), onde a alimentação concentrada correspondeu a $14,74 \%$ da composição do custeio. Todavia, inferiores aqueles encontrados por Lopes et al. (2009), onde a alimentação correspondeu a 59,95\% do custeio.

Com relação à suplementação volumosa que representou 7,98 $\pm 4,92 \%$ com coeficiente de variação de $61,7 \%$ na composição do custeio, estão representados os valores com cana-de-açúcar, capim picado, silagem e cevada disponibilizadas na região com baixo custo aos produtores. Em relação às despesas diversas, a composição no custeio foi de $4,79 \pm 3,05 \%$ com coeficiente de variação de $63,6 \%$. Sendo incluídas despesas de custeio com fretes do leite, combustível entre outros. 
Tabela 10. Composição das despesas de custeio: pastagem (Past.), alimentação concentrada mais polpa cítrica (A.C.), suplementação volumosa (S.V.), sal mineral (S.M.), medicamentos veterinários (M.V.), mão de obra (M.O.), assistência técnica (A.T.), luz e telefone (L.T.) despesas diversas (D.D.) e reparos e manutenção (R.M.) de 20 propriedades leiteiras na região Norte Fluminense, em porcentagem

\begin{tabular}{lcccccccccc}
\hline Propriedade & Past. & A.C. & S.V. & S.M. & M.V. & M.O. & A.T. & L.T. & D.D. & R.M. \\
\hline 01 & 38,81 & 8,43 & 6,57 & 1,56 & 4,52 & 25,11 & 2,48 & 2,15 & 8,35 & 2,02 \\
02 & 20,38 & 29,41 & 12,45 & 1,43 & 1,92 & 30,73 & 0,87 & 1,25 & 0,85 & 0,72 \\
03 & 25,39 & 8,22 & 2,60 & 2,92 & 1,93 & 48,54 & 4,29 & 1,92 & 1,92 & 2,28 \\
04 & 19,64 & 17,23 & 4,87 & 2,29 & 5,15 & 48,18 & 0,65 & 0,43 & 0,54 & 1,03 \\
05 & 17,46 & 24,01 & 7,76 & 2,04 & 2,77 & 35,16 & 2,22 & 2,46 & 2,35 & 3,76 \\
06 & 34,65 & 5,53 & 10,11 & 2,03 & 1,36 & 34,48 & 1,70 & 2,79 & 5,65 & 1,70 \\
07 & 28,05 & 13,22 & 7,66 & 1,56 & 4,71 & 25,49 & 4,42 & 1,78 & 9,84 & 3,27 \\
08 & 34,19 & 15,66 & 5,03 & 1,87 & 1,89 & 28,56 & 1,52 & 5,37 & 4,02 & 1,90 \\
09 & 30,29 & 6,79 & 2,80 & 1,46 & 1,52 & 46,68 & 2,18 & 3,83 & 2,72 & 1,73 \\
10 & 41,32 & 12,28 & 4,17 & 2,96 & 3,80 & 24,66 & 1,53 & 3,88 & 2,96 & 2,43 \\
11 & 37,77 & 15,34 & 9,75 & 3,61 & 2,66 & 20,98 & 1,27 & 2,26 & 5,27 & 1,08 \\
12 & 27,33 & 10,71 & 3,24 & 1,95 & 5,50 & 34,06 & 2,39 & 3,79 & 7,78 & 3,25 \\
13 & 48,15 & 3,96 & 5,51 & 2,43 & 3,71 & 24,68 & 1,50 & 2,71 & 6,66 & 0,68 \\
14 & 9,21 & 10,96 & 11,65 & 4,48 & 4,53 & 41,32 & 3,19 & 5,34 & 6,65 & 2,67 \\
15 & 25,11 & 9,75 & 8,33 & 3,65 & 6,52 & 32,81 & 1,38 & 1,87 & 8,46 & 2,13 \\
16 & 26,37 & 14,63 & 5,52 & 2,58 & 3,11 & 33,92 & 1,70 & 2,88 & 5,88 & 3,42 \\
17 & 30,14 & 7,04 & 11,25 & 0,67 & 2,74 & 29,79 & 2,02 & 1,97 & 9,97 & 4,41 \\
18 & 7,86 & 38,30 & 18,31 & 0,86 & 2,51 & 24,71 & 0,67 & 1,69 & 1,69 & 3,39 \\
19 & 5,24 & 22,85 & 19,86 & 1,18 & 2,51 & 40,31 & 1,86 & 2,82 & 1,61 & 1,76 \\
20 & 30,32 & 13,55 & 2,10 & 0,92 & 1,34 & 43,33 & 1,01 & 2,06 & 2,73 & 2,62 \\
\hline Média & 26,88 & 14,39 & 7,98 & 2,12 & 3,24 & 33,68 & 1,94 & 2,66 & 4,79 & 2,31 \\
DP & 11,24 & 8,58 & 4,92 & 1,01 & 1,50 & 8,57 & 1,04 & 1,25 & 3,05 & 1,04 \\
CV & 41,8 & 59,6 & 61,7 & 47,7 & 46,4 & 25,4 & 53,6 & 47,1 & 63,6 & 45,0 \\
\hline & & & & & & & & &
\end{tabular}

Com relação aos medicamentos veterinários foram encontrados valores médios de 3,24 $\pm 1,50 \%$ com coeficiente de variação de $46,4 \%$, semelhantes àqueles encontrados por Lopes et al. (2009) que obteve média de 3,76\% do custeio, diferente de Lopes \& Lopes (2001) e de Almeida Júnior et al. (2002) que encontraram médias de $6,29 \%$ e $5,62 \%$ respectivamente. Os demais itens como luz e telefone $(2,66$ $\pm 1,25 \%$ com coeficiente de variação de $47,1 \%)$, reparos e manutenção $(2,31 \pm 1,04 \%$ com coeficiente de variação de $45,0 \%)$, sal mineral $(2,12 \pm 1,01 \%$ com coeficiente de variação de $47,7 \%)$ e assistência técnica $(1,94 \pm 1,04 \%$ com coeficiente de variação de 53,6\%), apresentaram-se com baixas participações no custeio.

A assistência técnica foi o item de menor participação no custeio devido à divisão do valor monetário de uma visita técnica por todos os produtores analisados. Devendo este item ser repensado quanto a sua importância na atividade, pois com assistência técnica de qualidade e mais presente nas propriedades, os produtores podem ter ajuda profissional para melhorar os sistemas de produção com obtenção de maiores resultados na atividade leiteira.

A produção de leite deve ser pensada como uma atividade em conjunto com diversos fatores, utilizando índices zootécnicos e indicadores de eficiência econômica para analisar os pontos da atividade que devem ser melhorados para aumentar a produção de leite e a qualidade.

A obtenção de baixos indicadores zootécnicos encontrados para estes rebanhos principalmente em relação às taxas de natalidade $(57,4 \pm 12,18 \%)$ e produção de leite $(6,1 \pm 2,58$ litros) (Tabela 1) estão diretamente ligados com a obtenção de maiores receitas para a atividade. Lopes et al. (2009), constataram em seus estudos que a obtenção de índices zootécnicos adequados na criação de bovinos de leite influencia na composição e evolução dos rebanhos, sendo a taxa de natalidade a maior responsável na evolução dos rebanhos, seguida pela idade ao primeiro parto, taxa de descarte e taxa de mortalidade. Prado et al. (1995) observaram prejuízos econômicos em propriedades que apresentavam bons níveis de 
produção e produtividade devido à despreocupação com os indicadores zootécnicos. Segundo Marion \& Segatti (2006), cada vez mais são necessárias estratégias de desenvolvimento de sistema de gestão de planejamento e de custos agropecuários adaptados às pequenas propriedades rurais, relacionadas à cadeia produtiva do leite favorecendo o produtor frente ao mercado interno com inserção no cenário econômico atual, destacando sua contribuição na formação de renda e na absorção de mão de obra utilizada.

\section{Conclusões}

Os produtores obtiveram margem bruta e margem líquida positivas, permanecendo no curto prazo, pois o custo operacional efetivo está sendo coberto juntamente com a depreciação na atividade com possibilidades de expansão na produção de leite.

O resultado foi negativo representando prejuízo na atividade devido ao alto valor imobilizado no período de um ano de observação, não sendo possível a recuperação do capital investido tendendo à descapitalização no longo prazo.

A maior participação na composição das receitas e nas despesas de investimento foi o valor imobilizado, quando contabilizado, e a venda do leite e a compra de animais respectivamente, quando não contabilizado o capital financeiro. Em relação às despesas com custeio, as maiores porcentagens foram obtidas para mão de obra, pastagem, alimentação concentrada e suplementação volumosa, respectivamente.

\section{Agradecimentos e financiamento}

A CAPES e aos professores do Instituto de Zootecnia da Universidade Federal Rural do Rio de Janeiro.

\section{Referências bibliográficas}

Aguiar, A. P. A. \& Almeida, B. H. P. J. F. (2002). Planejamento e administração da produção de leite e carne no Brasil. Uberaba, Minas Gerais, Brasil: FAZU.

Almeida Júnior, G. A., Lopes, M. A. \& Pinatto, F. (2002). Efeito da venda de animais na rentabilidade de um sistema intensivo de produção de leite tipo B no estado de São Paulo. Reunião Anual da Sociedade Brasileira de Zootecnia, 391-4.

Bueno, A. A. O. (2013). Avaliação de sistemas de produção de leite em pastagens. $\mathrm{PhD}$, Universidade Estadual de Londrina, Londrina, Paraná, Brasil.

Bueno, P. R. B., Rorato, P. R. N., Dürr, J. W. \& Krug, E. E. B. (2004). Valor econômico para componentes do leite no estado do Rio Grande do Sul. Revista Brasileira de Zootecnia, 33(6):22562265.

Deresz, F. (1994). Manejo de pastagem de capim-elefante para produção de leite e carne. Simpósio sobre Capim-Elefante, 2116-137.

FAERJ/SEBRAE. (2010). Diagnóstico da Cadeia produtiva do leite do estado do Rio de Janeiro. Rio de Janeiro: FAERJ/SEBRAE-RJ.

Ferreira, A. M. (2002). Como reduzir o intervalo entre partos. DBO Especial Mundo do Leite, 132-33.

Godinho, R. F. \& Carvalho, R. d. C. R. (2013). Gestão de sistemas de produção de leite. Ciência et Praxis, 2(3):77-82.

Gomes, A. P. \& Alves, E. (1999). Identificando ineficiências na produção de leite. Boletim do Leite, 6(66):1-2.

Gonçalves, L. M. (2013). Análise dos custos de produção de leite em sistemas de capim-mombaça com diferentes fontes de suplementação volumosa. Monografia, Universidade Federal Fluminense, Rio de Janeiro, Brasil.

Lopes, M. A. \& Carvalho, F. M. (2000). Custo de produção do leite. Boletim Agropecuário, 321-42.

Lopes, M. A., Dias, A. S., Carvalho, F. M., Lima, A. L. R., Cardoso, M. G. \& Carmo, E. A. (2009). Resultados econômicos de sistemas de produção de leite com diferentes níveis tecnológicos na região 
de Lavras, MG nos anos 2004 e 2005. Ciência e Agrotecnologia, 33(1):252-260. doi: http://dix.doi.org/10.1590/S1413-70542009000100035.

Lopes, M. A., Lima, A. L. R., Carvalho, F. M., Reis, R. P., Santos, I. C. \& Saraiva, F. H. (2005). Resultados econômicos de sistemas de produção de leite com diferentes níveis tecnológicos na região de Lavras, MG. Arquivo Brasileiro de Medicina Veterinária e Zootecnia, 57485-493. doi: http://dix.doi.org/10.1590/S0102-09352005000400009.

Lopes, M. A., Lima, A. L. R., Carvalho, F. M., Reis, R. P., Santos, I. C. \& Saraiva, F. H. (2006). Efeito da escala de produção nos resultados econômicos de sistemas de produção de leite na região de Lavras (MG): um estudo multicasos. Boletim da Indústria Animal, 63(3):177-188.

Lopes, M. A., Lima, A. L. R., Carvalho, F. M., Reis, R. P., Santos, Í. C. \& Saraiva, F. H. (2004). Controle gerencial e estudo da rentabilidade de sistemas de produção de leite na região de Lavras (MG). Revista Ciência e Agrotecnologia, 28883-892.

Lopes, M. A. \& Lopes, F. M. (2001). Custo de produção da pecuária e análise de rentabilidade da pecuária leiteira. Paper presented at the Simpósio goiano sobre manejo e nutrição de bovinos, Goiânia, Goiás, Brasil.

Madalena, F. E., Matos, L. L. \& Holanda, J. R. (2001). A cadeia do leite no Brasil Produção de leite e sociedade (pp. 1-26). Belo Horizonte, Minas Gerais, Brasil: FEPMVZ.

Marion, J. C. \& Segatti, S. (2006). Sistema de gestão de custos nas pequenas propriedades leiteiras. Custose @gronegócios, 2(2):2-7.

Martins, P. C. (2004). Políticas públicas e mercados deprimem o resultado do sistema agroindustrial do leite. Juiz de Fora, Minas Gerais, Brasil: Embrapa.

Pereira, E. S., Pimentel, P. G., Queiroz, A. C. \& Mizubuti, I. Y. (2010). Novilhas leiteiras (Vol. 1). Fortaleza, Ceará: Graphiti Gráfica e Editora Ltda.

Pereira, P. A. C., Ferreira, A. M. \& Carvalho, L. B. (2009). Perfil sorológico das leptospiroses em rebanhos bovinos leiteiros da região do Vale do Paraíba-SP. Revista Brasileira de Reprodução Animal, 28(3):174-176.

Prado, E., Cruz, F. E., Vianna, F. C., Torres, A. M. \& Reis, D. L. (1995). Avaliaçäo do desempenho técnico econômico de exploraçöes leiteiras em Divinópolis-MG, segundo a forma de produçäo. Arquivo Brasileiro de Medecina Veterinária e Zootecnia, 47(3):361-372.

Prado, E., Geraldo, L. G. \& Cardoso, B. M. (2007). Rentabilidade da exploração leiteira em uma propriedade durante cinco anos. Arquivo Brasileiro de Medicina Veterinária e Zootecnia, 59(2):501-507.

Robbs, P. G. \& Campelo, J. C. F. (2002). Produção segura na cadeia do leite. Segurança Alimentar na Cadeia do Leite, 1(1):51-76.

Rocha, A.C.S.C. 2013. Análise de custo de produção para novilhas leiteiras em pastagem de capim-xaraés suplementadas com mistura mineral. Monografia (Engenharia de Agronegócios). UFF.

Silva, H. A., Moraes, H. S. K. A., Hack, V. A. G. E. \& Faccio, P. C. F. (2008). Análise da viabilidade econômica da produção de leite a pasto e com suplementos na região dos Campos Gerais-Paraná. Ciência Rural, 38(2):445-450.

Recebido: 18 de agosto, 2019.

Aprovado: 15 de outubro, 2019.

Publicado: 30 de novembro, 2019.

Licenciamento: Este artigo é publicado na modalidade Acesso Aberto sob a licença Creative Commons Atribuição 4.0 (CC-BY 4.0), a qual permite uso irrestrito, distribuição, reprodução em qualquer meio, desde que o autor e a fonte sejam devidamente creditados. 